\title{
EFFECT OF TAPERED INTERFERENCE FIT BETWEEN IMPELLER AND SHAFT IN TURBO MACHINES
}

\author{
NILESH Vishwakarma ${ }^{1}$, AVINASH Renuke ${ }^{2}$, V.M. Phalle ${ }^{3}$ \\ ${ }^{1}$ Department of Mechanical Engineering, Veermata Jijabai Technological Institute, Mumbai-400019, India, \\ e - mail: nilesh.vishwakarmaaa@gmail.com \\ ${ }^{2}$ Design and NPI Engineer, GE Oil \& Gas, Bangalore-560066, India, e - mail: avinash.renuke@ge.com \\ ${ }^{3}$ Faculty of Mechanical Engineering, Veermata Jijabai Technological Institute, Mumbai-400019, India, \\ e-mail:vmphalle@vjti.org.in
}

\begin{abstract}
In Turbo machines, there are many components which are provided with interference to transmit torque and to maintain contact between them. There are transient scenarios where impeller and shaft lose interference and limits the speed of the impeller. In this paper, a case study on centrifugal compressor has been presented to see the effect of taper pairing of shaft with impeller. When the centrifugal compressor starts then a transient condition is dominant wherein the impeller of the centrifugal compressor gains higher temperature quickly compared to shaft. Since the thermal expansion of the impeller will be much more than the shaft, interference between impeller and shaft is likely to reduce. With reduced interference, the impeller should retain minimum required contact pressure with the shaft and the torque transmitting capability. For conventional fitted impeller, stress built up is a major concern especially at higher speeds. This paper gives a proposal about the importance of tapered interference fit method compared to cylindrical fitted impeller assembly. An effective analysis between the conventional straight fitted impeller and impeller having tapered bore is presented for the same torque transfer capability. The analysis reveals that for the impeller with tapered bore, the stress for impeller reduces and a healthy contact pressure exists.
\end{abstract}

KEYWORDS: taper, turbo machine, compressor, impeller, centrifugal compressor, taper interference fit, straight fit, taper fit, contact pressure, equivalent stress

\section{Introduction}

In turbo machineries, particularly the connection between the shaft and impeller or rotor or disc needs to be securely mounted on the shaft with due care especially at higher speeds. The assembly between impeller-shaft should be such that the impeller must retain contact with the shaft for all times of operation.

Various methods are available to assemble impeller; however, emphasis should be made for best design constraints. Key is one of those methods to establish connection between the components to be assembled. On the other hand, impellers are shrink-fitted due to which the torque transfer is solely dependent on how good the interference is available between impeller and shaft. Use of key in centrifugal compressor is liable to induce stress concentration; hence efforts should be made to assemble the impeller with shaft by maintaining good interference between them to succeed without using key. Healthy contact pressure helps us in evaluating the keyless condition. Another method similar to key is the use of spline. Splines are series of axial keys machined into a shaft, with corresponding grooves machined into the bore of the mating part. It has higher load carrying capacity and it can transfer axial, rotational and torsional loads; however, surface wear, fretting corrosion fatigue, tooth failures are the common failure modes. A novel method to assemble impeller and shaft in centrifugal compressor developed by David B. Herrick [1], the impeller includes a hub portion having each end provided with a plurality of radially spaced axially extending slots. In this method as the compressor speed increases the 
end portions of the hub are biased into tighter gripping engagement with the shaft preventing radial movement of the impeller relative to shaft. Creating the slot may lead to strength concern of the components being assembled. A composite shaft-disc work piece which is assembled from a disc with a passage hole and from a shaft employs friction welding method [2]. When a healthy contact status is desired between the components to be assembled then shrink fit is the most preferred method to employ interference fit. In this method the hub is heated to a suitable temperature and then it is assembled on the shaft. After cooling of hub, the interference fit is created. Similarly, the shaft can be cooled and assembled with hub to create interference fit after reaching room temperature. A research study on press-fit evaluation and study of displacement in temperature changes helps to determine optimal radial clearance [3].

Normally, conventional cylindrical method employing shrink fit is used to assemble impeller and shaft; however, stress built up in impeller is a major concern besides residual stresses. Having conferred to various methods and their limitations, taper fit method helps to achieve better results such as reduced stress levels, improved contact status and contact pressure, better torque transfer capability. An attempt is made to analyse the effect of tapered interference fit between impeller and shaft with respect to conventional cylindrical fit method to have better understanding of the performance.

\section{Literature review}

An innovative idea presented by L. Haugen, Robert King and Jeff Schmidt on Tapered Polygon Coupling for impeller [4], the shaft has a tapered bore having a polygonal crosssection. The impeller includes a corresponding tapered polygon plug configured to be placed in the bore of the shaft. Additionally, the impeller may include the tapered bore having a polygonal cross-section, and the shaft may include the tapered polygon plug configured to be placed in the bore of the impeller. The plug of the shaft is split so that when the fastener is inserted into the passage the plug expands against the side wall of the bore thereby creating an interference fit between the shaft and the impeller. This method gives an idea that the taper fit can be employed for coupling or assembly of components.

The tapered interference fit, and its contact stress induce more and more relative study work. Stress variations of various configurations of the taper lock used to connect the turbine shaft to the turbine disk and when used in belt-conveyor systems are carried by V. Ramamurti and R. Karthikeyan [5]. Also, the effect of structures and press forces on the contact stress were studied and gives useful information [6,7].

The mechanics of the tapered interference fits in dental implants and the analysis carried by Dincer Bozkay and Sinan Mueftue provides a reliable connection in addition to the stability of the implant-abutment. The analysis shows that plastic deformation in the implant limits the increase in the pull-out force that would have been otherwise predicted by higher interference values [8].

Tapered interference fits can avoid the influence of keyways on the parts strength and transfer high torques. The study done on the influence of taper on the interference fit between a propeller hub and a shaft reveals improved stress distribution of the propeller hub and an effective approach to increase the connection strength [9]. For evaluating the quality of interference fits an improved design method utilizes safety factor for assuring component strength and factor of safety for transmitting desired power. Employing maximum and minimum interference limit provides a quantitative guideline for interference fit design [10]. Also, the pressure generated at the contact surfaces of two axisymmetrical components assembled together with an interference fit, can be evaluated [11]. 
Tapered joint can be considered as a power transmission component which transfers rotational power. Tapered joint hardly induces any stress concentration because of no use of key or cotter to prevent rotational slip in addition to advantage of ease in assembly or disassembly. In a centrifugal compressor if the stresses are reduced then the load carrying capacity can be increased. The compressor would be able to run at higher speed to meet the pressure requirements. A higher interference fit may induce defects in the form of fatigue cracks occurring in load-carrying section and affects the ability of a structural component to transfer the required loads, which may result in structural component damage [12].

Generally, conventional equations are used for stress calculations in interference-fit designs. However, if the component shows elastic-plastic behavior the process becomes more complex. When the centrifugal compressor starts then the impeller gains higher temperature swiftly and shaft remains in the vicinity of ambient temperature. This leads to transient state of operation, wherein the boundary conditions of the system are changed continuously. In such interference fitted connections the occurrence of plastic deformation zone and its distribution study becomes dominant [13]. Taper fit plays an important role to serve the purpose especially in transient condition to take care for higher stresses developed in conventional shrink fitted impellers.

\section{$3 \quad$ Nomenclature}
E Young's Modulus of Elasticity (MPa)
St tangential stress developed between hub and shaft (MPa)
$\mathrm{Sr}$ radial stress developed between hub and shaft (MPa)
$\Delta \mathrm{D}$ change in inner diameter of hole $(\mathrm{mm})$
a internal radius of the collar $(\mathrm{mm})$
b external radius of the collar ( $\mathrm{mm})$

\section{$4 \quad$ Modelling}

A suitable sized 2D impeller for straight and taper fitted assembly as shown in Fig.1 is modeled using $\mathrm{NX}^{\mathrm{TM}}$ software. Fig.1A represents the straight or conventional fitted impeller assembly and Fig.1B depicts the tapered fitted impeller assembly. Appropriate taper size is chosen for analysis [14]. The following basic geometry is used for analysis:

Impeller Diameter: $390 \mathrm{~mm}$

Impeller bore: $146 \mathrm{~mm}$ (tolerance: $\pm 0.1 \%$ )

Impeller bore length: $48 \mathrm{~mm}$

Interference: $0.1402 \mathrm{~mm}$

Shaft diameter: $146 \mathrm{~mm}$ (tolerance: $\pm 0.1 \%$ )

\section{Analysis}

ANSYS ${ }^{\circledR}$ software package is used for analysis. Static structural analysis with no temperature load is carried out in workbench. Also, standard GE tool is used for validation. The following material properties were used for analysis:

Impeller material properties:

Material Density - $7850 \mathrm{~kg} / \mathrm{m}^{3}$

Poisson Ratio - 0.3

Young's Modulus - $196500 \mathrm{MPa}$ 
Shaft material properties:

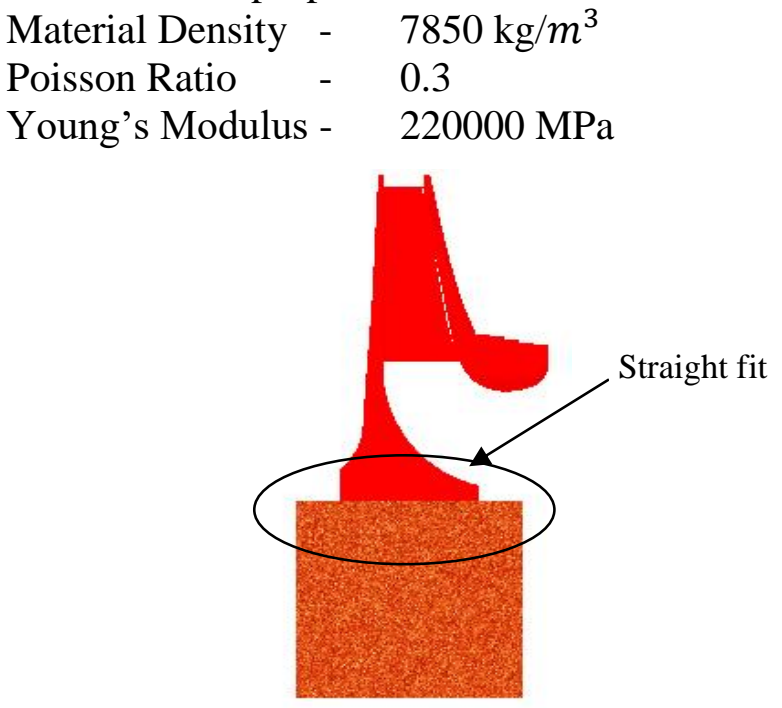

$(1 \mathrm{~A})$

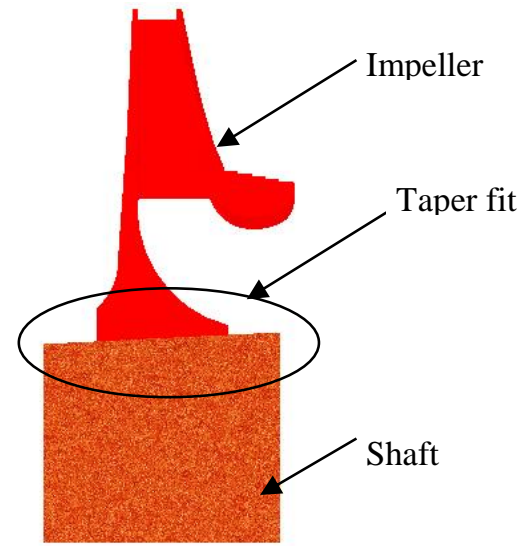

(1B)

Fig. 1 Impeller-shaft assembly, 1A. Straight fitted impeller, 1B. Taper fitted impeller

For swift response a slice model is considered for analysis. The straight fit slice model as shown in Fig.1A is seen in static structural environment. Contact constraints with respect to shaft and impeller besides uniform geometric interference is applied using add offset, rammed effects. Cyclic symmetry is applied so that the program solves for the full cyclically symmetric model using the basic sector model as shown in Fig.2B.

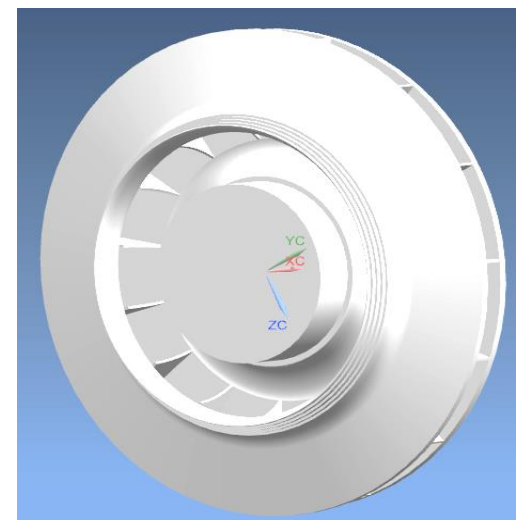

(2A)

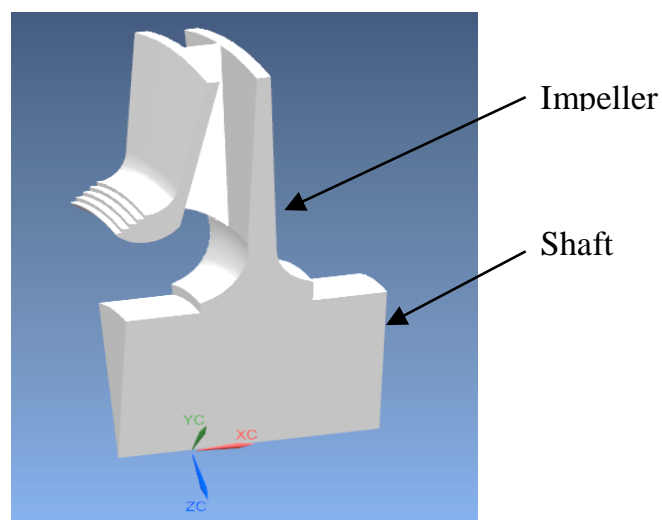

(2B)

Fig. 2 Full model and slice taper model, 2A. Full model, 2B. Slice taper model

Standard fine meshing is done for the impeller assembly considering the accuracy and contact constraints besides uniform geometric interference for straight fit and tapered fit is applied. Fig.2B depicts the impeller assembly slice having tapered geometry. After imposing proper boundary conditions and applied loadings the model is solved for straight and taper fitted impeller respectively. After solution, equivalent Von-Mises stress and contact pressure are analyzed. The flowchart shown in Fig.3 presents the steps followed for analysis.

\section{$6 \quad$ Results \& Discussion}

The principal focus is to analyze the equivalent Von-Mises stress for the impeller and contact pressure available between shaft and impeller for safe operations. Lower stress and better contact pressure is the deciding criteria. 


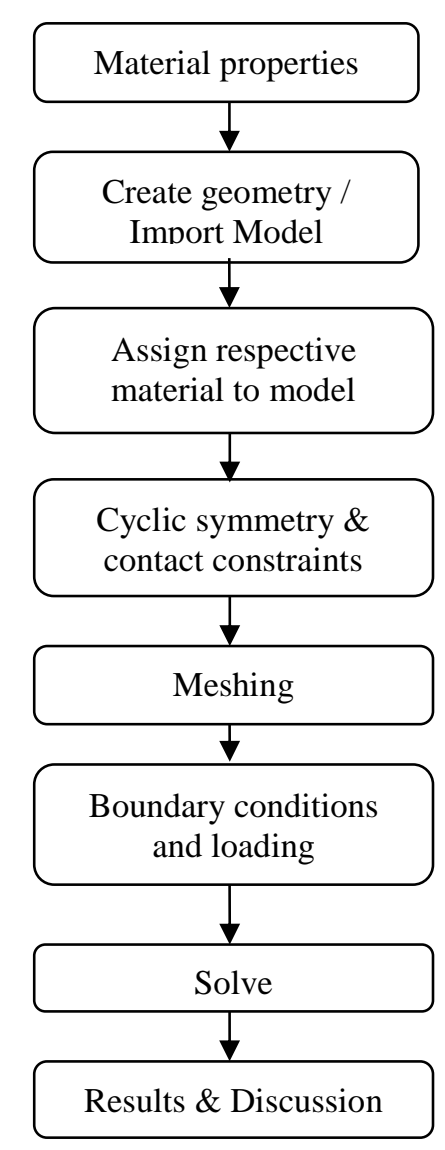

Fig. 3 Steps for analysis

When the contraction of the outer hole or impeller bore on the shaft has occurred then the amount of holding stress will be developed [15]. This holding stress refers to tangential stress or circumferential stress or bore hoop stress (1) and radial stress (2).

$$
\begin{aligned}
& \mathrm{St}=\frac{\mathrm{E} \times \Delta \mathrm{D}}{4 \times \mathrm{a}}\left(1+\frac{\mathrm{a}^{2}}{\mathrm{~b}^{2}}\right) \\
& \mathrm{Sr}=\frac{\mathrm{E} \times \Delta \mathrm{D}}{4 \times \mathrm{a}}\left(1-\frac{\mathrm{a}^{2}}{\mathrm{~b}^{2}}\right)
\end{aligned}
$$

Since centrifugal impellers run at high speed, an attempt is made to analyze the stress at different speed levels i.e. the maximum continuous speed $(300 \mathrm{~m} / \mathrm{s})$, the trip speed $(330 \mathrm{~m} / \mathrm{s})$ or rotor over-speed and the impeller over-speed $(345 \mathrm{~m} / \mathrm{s})$ for $200 \mathrm{~kW}$ power. Usually at overspeed, the impeller is analyzed in absence of shaft and only speed is applied to the impeller in addition to degree of freedom constraints.

From the Table1, it can be inferred that the stresses are less for the tapered fit impeller as compared with straight fitted impeller assembly i.e. the stress reduction for maximum continuous speed is around $12.5 \%$ and likewise the decreased stress values are seen for trip speed and impeller over-speed besides contact pressure being at par with straight fit impeller assembly.

Figure 4 shows the von-mises stress for trip speed or rotor over-speed for conventional interference fit and taper fit impeller assembly. It is observed that the stress levels for taper fit are lower comparatively. Also, considering the fillet zone for hub and shroud with respect to 
blade besides the region in the vicinity of the bore, lesser stresses are observed compared to global stress variations for conventional fitted impeller.

Table. 1 conventional and tapered interference fit results

\begin{tabular}{|l|c|c|c|}
\hline Results & $\begin{array}{c}\text { Conventional Fitted } \\
\text { Impeller } \\
\text { (cylindrical) }\end{array}$ & $\begin{array}{c}\text { Taper Fitted } \\
\text { Impeller }\end{array}$ & $\begin{array}{c}\text { Stress Reduction } \\
\text { for Taper Fit (\%) }\end{array}$ \\
\hline Stress at impeller over-speed (MPa) & 949.85 & 890.11 & 6.29 \\
\hline Stress at rotor over-speed (MPa) & 932.88 & 858.24 & 7.91 \\
\hline Stress at Maximum Continuous Speed (MPa) & 857.23 & 749.86 & 12.52 \\
\hline Average Contact Pressure (MPa) & 208.91 & \multicolumn{2}{|c|}{162.03} \\
\hline
\end{tabular}

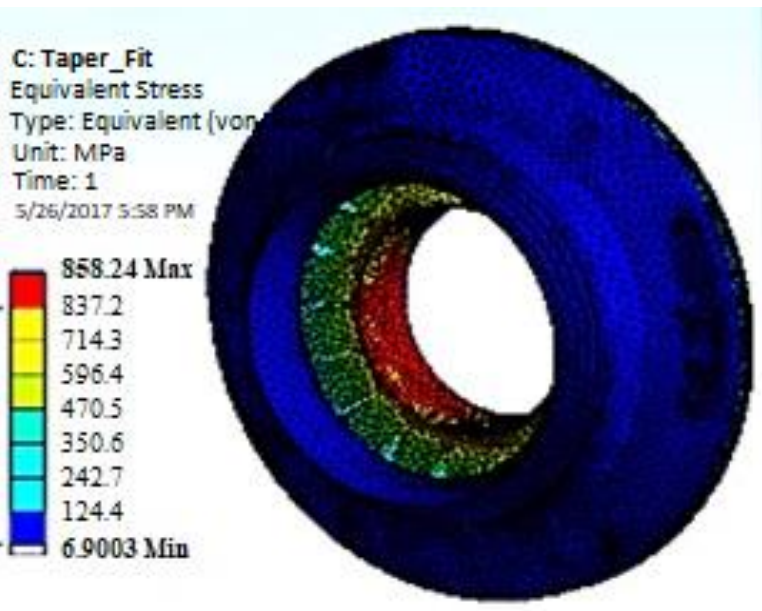

(A)

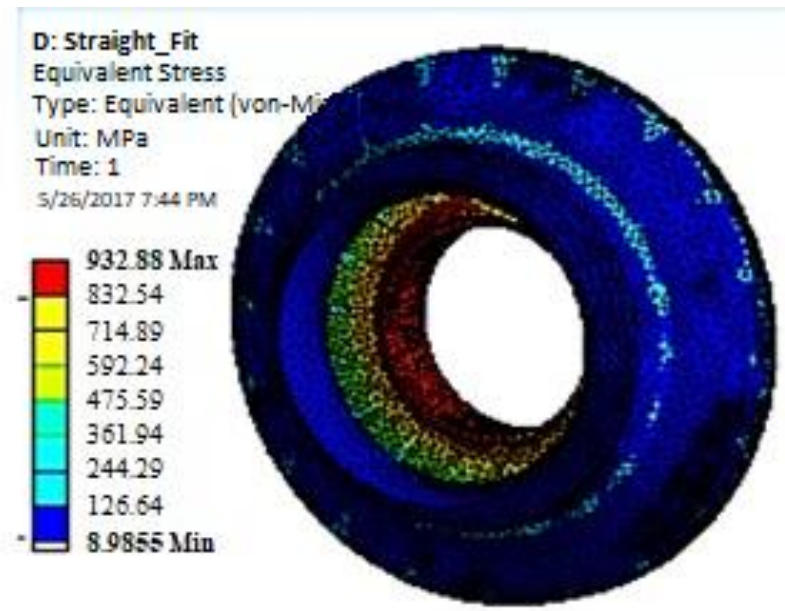

(B)

Fig. 4 Von-mises stress at trip speed, A. Taper Fit, B. Straight Fit

Figure 5 shows the linearized equivalent stress along the bore length for impeller over-speed. It is observed that the maximum stress is $604.98 \mathrm{MPa}$ along the bore length. The stresses are less along the bore length. The higher stress as observed from Fig.4 and Table1 are the confined to a small area i.e. localized stresses, which generally occurs at the region pertaining to hub and shroud with respect to fillets. These higher stresses can be controlled by improving the fillet radius.

Figure 6 shows the contact pressure trend along the impeller bore length which is almost similar for tapered interference fit and conventional interference fit. The average contact pressure of $162.03 \mathrm{MPa}$ for taper fit from Table1 shows healthy bond between impeller and shaft. With increase in contact pressure, fretting is reduced [16]. It is conferred from Fig.6 that for the taper interference fit, the contact pressure can be achieved well to suit the design constraints for improved performance.

Also, as seen in the Fig.7, the contact status for taper fit confirms stickiness for the major region of impeller bore length which reveals healthy connection between impeller and shaft assembly. Better contact status helps to retain the impeller firmly with the shaft, thereby maintaining good interference fit in addition to provide the benefit of keyless condition.

\section{CONCLUSION}

The analysis of the conventional cylindrical method and the tapered interference fit shows that the stress levels for taper fit can be reduced in comparison with cylindrical approach. Also, the taper interference method of assembling the impeller and shaft maintains healthier contact status and contact pressure at par with conventional fit assembly as confirmed by the analysis 
of impeller of centrifugal compressor. Healthy contact pressure helps the impeller to run in keyless condition thereby taking care of stress concentration. It can be inferred that if the stresses are reduced besides improved contact pressure then it is possible for turbo machines to sustain higher loads and better torque carrying capability. With the above results and discussions, it can be concluded that tapered interference fit helps to achieve better results.

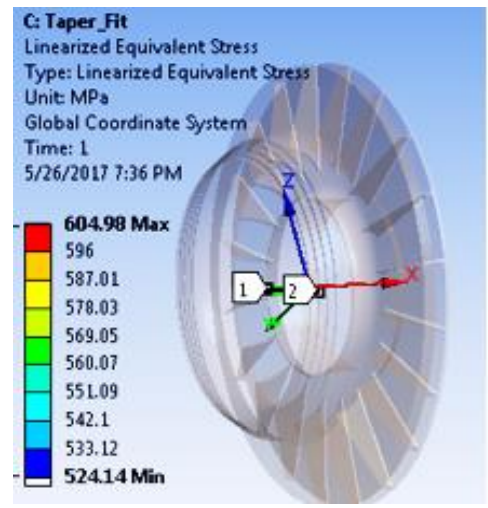

\section{Linearized equivalent stress along the impeller bore length}

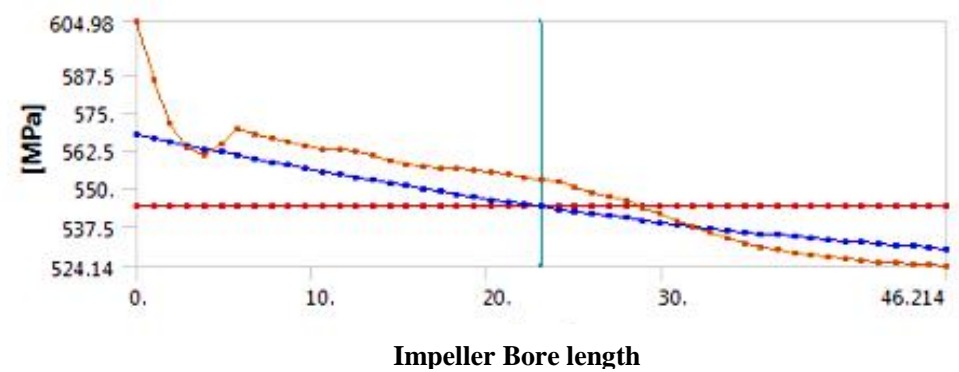

Stress along bore length

Stress along bore thickness

$\ldots$ Average stress along bore length

Fig. 5 Linearized equivalent stress along the bore length (line 1-2)
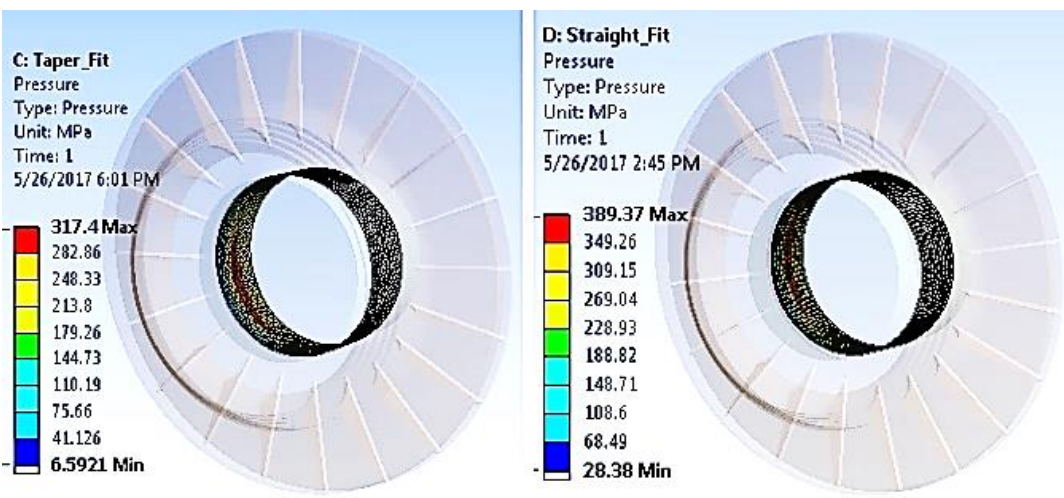

Fig. 6 Contact Pressure - Taper Fit and Straight Fit
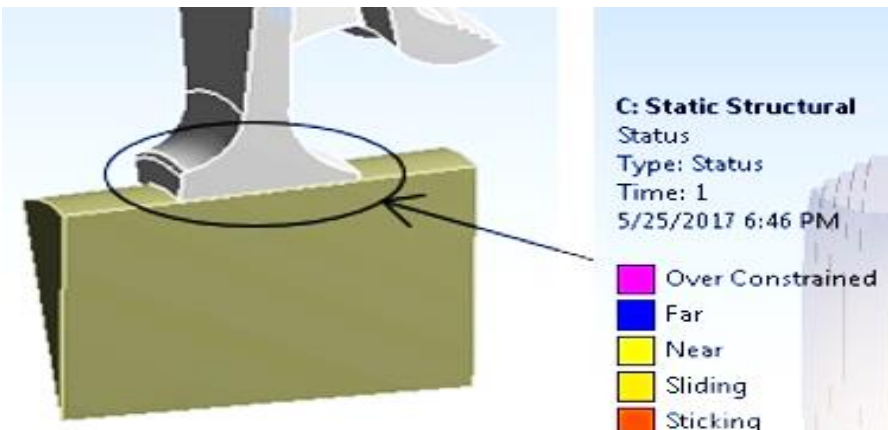

Fig. 7 Contact status along bore length for taper fit 


\section{ACKNOWLEDGEMENT}

I am extremely grateful to GE Oil \& Gas for giving me the opportunity to do internship and would like to extend the gratitude to my mentors at the organization and the institution which has driven me towards research activities and to come up with, out of box thinking ideas besides emphasizing the zeal for learning.

\section{REFERENCES}

[1] David B. Herrick. Impeller and Shaft Assembly, International patent application no. US3884595, 1975.

[2] J. Bender, C. Hentrich, J. Koll, T. Mayer, R. Reinhardt, H. Zechmann, R. Paasch. Friction-welded shaft-disc assembly and method for the manufacture thereof. International patent application no. US6660407, 2003.

[3] P. Šlesar, R. Jančo. Press-fit evaluation and study of displacement in temperature changes. Journal of Mechanical Engineering - Strojnícky časopis, 2018 (68), 103 - 108.

[4] Ronald L. Haugen, Robert King, Jeff Schmidt. Tapered Polygon Coupling. International patent application no. US 0164252, 2002.

[5] V. Ramamurti, R. Karthikeyan. Stress analysis of taper locks. Computers \& structures 1997 (62), $721-736$.

[6] A. Ozel, S. Temiz, M. D. Aydin, Sadri Sen. Stress analysis of shrink-fitted joints for various fit forms via finite element method. Materials \& design 2005 (26), 281 - 289.

[7] I. T. Cook, H. Fessler, T. H. Hyde, N. A. Warrior. Assembly stresses in taper - locking shaft couplings Part 1: Photoelastic work. Journal of Strain Analysis for Engineering Design 2001 (1), 25 - 34.

[8] D. Bozkay, S. Muftu. Mechanics of the tapered interference fit in dental implants. Journal of Biomechanics 2003 (11), 1649 - 1658.

[9] X. Q. Zhao, P. Shang. Exact Solution of Stresses of Tapered Interference Fit. Applied Mechanics and Materials 2014 (556 - 562), 4284 - 4287.

[10] Y. Zhang, B. McClain, X. D. Fang. Design of interference fits via finite element method. International Journal of Mechanical Sciences 2000 (42), 1835 - 1850.

[11] B. Parsons, E. A. Wilson. A method for determining the surface contact stresses resulting from interference fits. Journal of Engineering for Industry 1970 (92), 208 - 218.

[12] R. Owsiński, A. Niesłony. Identification of fatigue cracks on the basis of measurable changes in system dynamics, Journal of Mechanical Engineering - Strojnicky časopis 2017 (67), $77-84$.

[13] S. Sen, B. Aksakal. Stress analysis of interference fitted shaft - hub system under transient heat transfer conditions, Journal of Materials \& Design 2003 (25), 407 - 417.

[14] V. Nilesh, V. M. Phalle. Analyze Different Taper Sized Assembly. International Research Journal of Engineering and Technology 2017 (4), 2990 - 2993.

[15] Edge, LLC. Engineers. Shrink Thermal Fit Review, Engineers Edge - Engineering and Manufacturing Solutions 2012.

[16] T. Juuma. Torsional fretting fatigue strength of a shrink-fitted shaft. Journal of Wear 1999 (231), $310-318$. 